Article

\title{
Construction Theory for a Building Intelligent Operation and Maintenance System Based on Digital Twins and Machine Learning
}

\author{
Yuhong Zhao ${ }^{1,2}$, Naiqiang Wang ${ }^{1,2}$, Zhansheng Liu ${ }^{1,2, *}$ and Enyi Mu ${ }^{3}$ \\ 1 College of Architecture, Civil and Transportation Engineering, Beijing University of Technology, \\ Beijing 100124, China; zhaoyuhong@bjut.edu.cn (Y.Z.); wangnq@emails.bjut.edu.cn (N.W.) \\ 2 The Key Laboratory of Urban Security and Disaster Engineering of the Ministry of Education, \\ Beijing University of Technology, Beijing 100124, China \\ 3 College of Urban and Environmental Sciences, Urban and Economic Geography, Peking University, \\ Beijing 100871, China; emu@stu.pku.edu.cn \\ * Correspondence: liuzhansheng@bjut.edu.cn
}

check for

updates

Citation: Zhao, Y.; Wang, N.; Liu, Z.; $\mathrm{Mu}, \mathrm{E}$. Construction Theory for a Building Intelligent Operation and Maintenance System Based on Digital Twins and Machine Learning. Buildings 2022, 12, 87. https:// doi.org/10.3390/buildings12020087

Academic Editors: Cynthia Changxin Wang, Brian Lee and Sara Shirowzhan

Received: 15 November 2021

Accepted: 16 January 2022

Published: 18 January 2022

Publisher's Note: MDPI stays neutral with regard to jurisdictional claims in published maps and institutional affiliations.

Copyright: () 2022 by the authors. Licensee MDPI, Basel, Switzerland. This article is an open access article distributed under the terms and conditions of the Creative Commons Attribution (CC BY) license (https:/ / creativecommons.org/licenses/by/ $4.0 /)$.

\begin{abstract}
The operation and maintenance (O\&M) of buildings plays an important role in ensuring that the buildings work normally, as well as reducing the damage caused by functional errors. There are obvious problems in the traditional O\&M modality, and an effective way to solve them is to make the model smarter. In this paper, a digital twin framework for building operation is proposed, which consists of two key components: a digital twin O\&M model and a machine learning algorithm. The process of establishing the digital twin model is introduced in detail, and the method is explained according to the structure, equipment, and energy consumption characteristics of the model. A mechanism of fusing the digital twin and machine learning algorithm is proposed and the prediction process based on an artificial neural network (ANN) is shown. Finally, based on a systematic summary of the modeling process and fusion mechanism, the development path and overall structure of the intelligent O\&M system utilizing digital twins is proposed.
\end{abstract}

Keywords: digital twin; machine learning; artificial neural network; operation and maintenance

\section{Introduction}

In the process of operating a building, it is difficult to maintain the structures at a safe status all the time, or even in a dangerous state, due to the degradation of materials and damage caused by various harmful factors [1]. Building maintenance has long been recognized globally as a long-term problem in the life cycle of buildings because of the increasing number of deteriorating buildings [2-4]. As a part of building operation, it is important to conduct maintenance work, such as repairing failures, replacing components, and discovering construction problems-and solving them in time to ensure normal and safe running status [5].

The traditional O\&M pattern of buildings has a series of problems, such as data management difficulties, poor measurement and detection accuracy, single technical means, and high cost [6]. In order to solve these problems and promote the development of O\&M technology, experts have conducted a great deal of research and investigations on intelligent O\&M management. Yu et al. [7] reported on the importance of BIM technology and analyzed the processes of a digital O\&M system. Li et al. [8] put forward a BIM-based construction O\&M system that realizes data visualization and improves efficiency. Taffese et al. [9] introduced Internet of Things (IoT) technology into the process of structurally intelligent O\&M and created a framework for monitoring the durability of concrete structures in order to improve the accuracy of structural state assessments. Shigeru Yasuda et al. [10] set up a data acquisition and transmission system and an automatic fatigue detection system by 
using IoT technology, and a cloud server system that enables users to observe the state of buildings from their office. Ramin et al. [11] used a wavelet pocket relative energy (WPRE), wavelet weighted least square support vector machine (WWLS-SVM), and other algorithms to monitor the health of a building's structure and reduce their data dimension. Gabriele et al. [12] observed elevator usage with eye-tracking technology, established an elevator operation simulation model, predicted elevator usage under different conditions, and optimized related operation measures.

The concept of digital twins has been evolving since its inception in 2002, generating multiple definitions and interpretations-some of them differing significantly [13-15]. In recent years, in order to better apply digital twin technology, the concept has been analyzed many times and some of the characteristics have been widely recognized [16-18]. Digital twins should be the most realistic virtual representation of physical entities, including the digital model and all relevant information and should be synchronized with those entities. Another characteristic of digital twins is self-evolution, which means that they need to change and evolve according to the actual situation while maintaining the contrast between physical and virtual spaces. Digital twins have a wide variety of applications in manufacturing industries [19]. Cai et al. [20] used digital twins in additive manufacturing with augmented reality, but there were inherent inaccuracies. Moretti et al. [21] combined geometry monitoring and process quality control for fused filament fabrication through digital twin and optical imaging, but it was limited to geometry. Compared with manufacturing, the construction industry is more complicated due to its wide range of fields and long production cycles. Therefore, the realization of digital twins is more difficult and there are fewer relevant results.

Digital twins have two key characteristics, interactive feedback and self-evolution, and can realize intuitive observations and predictions of the working state, which makes operation and maintenance work more efficient, timely, and intelligent [22]. Leser et al. [23] applied digital twin technology to the health management of fatigue-critical structures and noted that it has application potential in the operation and maintenance processes of multiple fields, from engineering to medical treatment. The paper only put forward ideas and did not give practical applications. Ye et al. [24] built a digital twin model for structural monitoring; however, the limitation of that study was that it did not apply digital twins to the overall O\&M. Sivalingam et al. [25] combined physics and digital twin technology to predict the working state and remaining life of offshore wind turbines. They researched equipment, which has different O\&M requirements compared to buildings. Liu et al. [26] proposed a dynamic guidance method for fire evacuation based on digital twins combined with a support vector machine and obtained a good evaluation and prediction model. However, the method is only meaningful for evacuation in the event of a fire, which is of little help with regard to the overall O\&M process.

In a word, there are few articles on applying digital twins to the full range of building O\&M. To solve the problems mentioned above and improve the intelligence level of the model, this paper emphasizes the advantages of digital twins and contributes to:

- Proposing a fusion of digital twins and machine learning driven by a method of predicting a building's O\&M status;

- Establishing an overall structure and development route for the O\&M platform based on digital twins and machine learning;

- Using software engineering technology to achieve building visualization, resource sharing, and simple and efficient operation.

The remainder of the paper is organized as follows. Section 2 proposes a framework and describes its composition and functions, with brief descriptions of the contents of Sections 3 and 4 . Section 3 describes in detail how to establish an O\&M model based on digital twins. Section 4 presents the fusion mechanism of digital twins and machine learning, which can predict status, and this method is proved to be feasible. Section 5 introduces how to build an O\&M system based on digital twins and introduces its composition. Section 6 discusses the results and concludes the paper. 


\section{Digital Twin Framework for Building Operations}

The characteristics of digital twin virtual interaction and real-time feedback are conducive to improving the informatization and automation of building O\&M management. Aiming at matching the characteristics and requirements of building O\&M governance, this paper pays attention to the critical problems of structure, equipment, and energy consumption. It establishes a digital twin multidimensional model for building operation and maintenance management [27]. In Equation (1), $M_{\mathrm{ODT}}$ is a digital twin model for building O\&M management, $B_{\mathrm{OPE}}$ is a physical building entity, $B_{\mathrm{OVE}}$ is a virtual building entity, $B_{\mathrm{ODD}}$ is the real-time data obtained by sensors and other devices in a running building, $B_{\mathrm{OPD}}$ is the predictive data obtained by the machine learning algorithm, and $B_{\mathrm{OCN}}$ is the connection between various parts:

$$
M_{\mathrm{ODT}}=\left(B_{\mathrm{OPE}}, B_{\mathrm{OVE}}, B_{\mathrm{ODD}}, B_{\mathrm{OPD}}, B_{\mathrm{OCN}}\right)
$$

According to the above model, and based on the characteristics and needs of the construction operation and maintenance process, a digital twin framework is proposed, as shown in Figure 1.

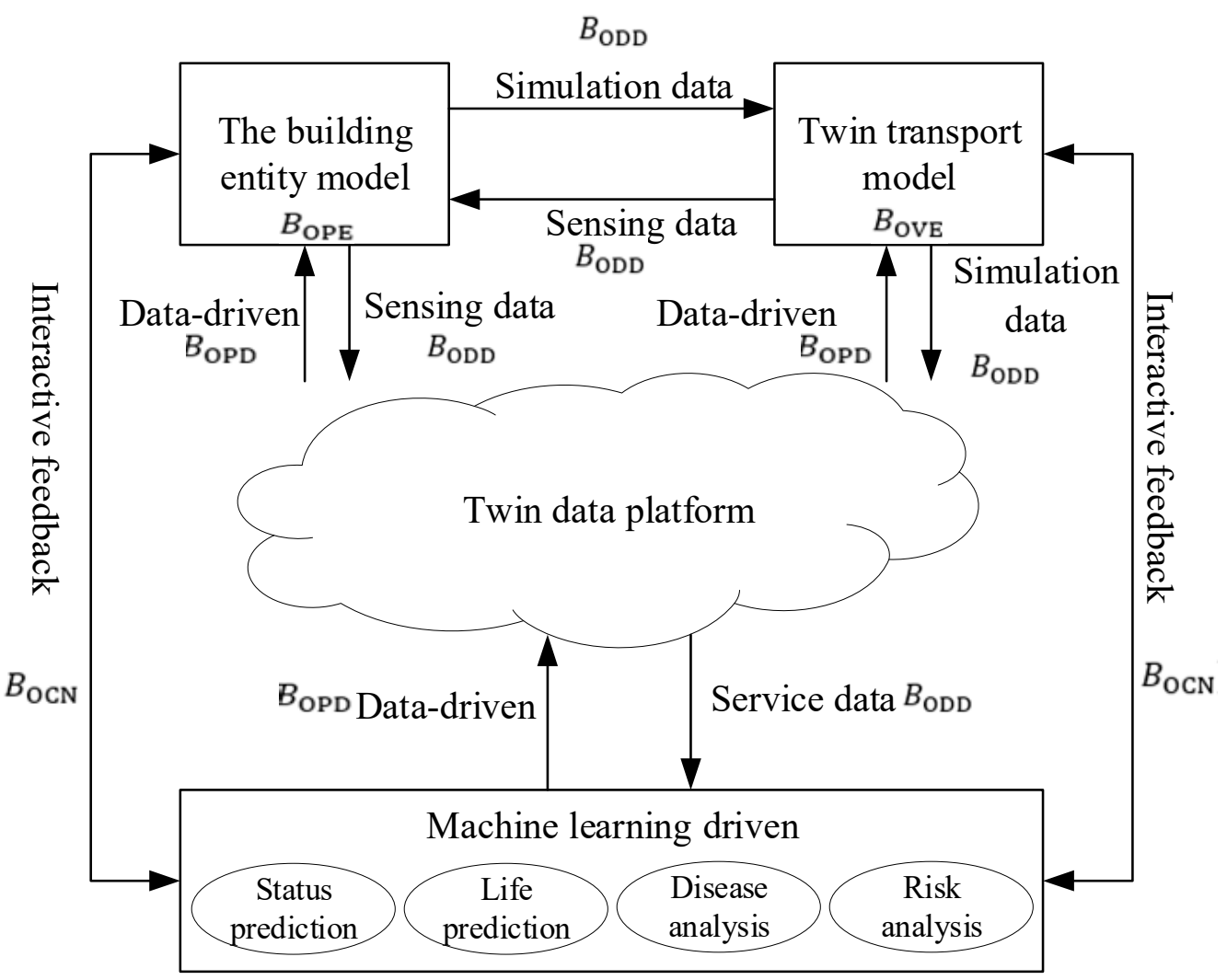

Figure 1. Digital twin framework for building operation and maintenance.

Based on the physical entity of the building and its BIM model, this framework, combined with emerging technologies—such as BIM, Internet of Things, and intelligent sensors-applies intelligent algorithms-such as neural networks and decision trees-to establish a digital twin operation model consisting of geometric, physical, and behavioral aspects and rules. The model is divided into digital twin operation models of building structure, building equipment, and building energy consumption [28]. The data between the digital twin O\&M model and the physical building entity interact in real time and are stored according to the O\&M model classification on the twin data platform. Machine learning algorithms are needed to drive the analysis and processing of the twin data platform. By analyzing the data, it can diagnose and predict the building status. State 
prediction is an important means to ensure the normal running of a building. Machine learning algorithms are more widely used in state prediction, including neural networks, support vector machines, and decision forests. Among them, an artificial neural network has better durability and timeliness [29]. It has a high degree of self-learning, adaptability, and error-proofing ability [30]. Appropriate network parameters can be determined to obtain the best value of the results [31]. This characteristic of artificial neural networks is suitable for the O\&M process of buildings.

The framework, based on the information contained in the twin transport model and driven by machine learning algorithms, realizes services such as predicting building condition and residual life, analyzing disease and risk, etc. Furthermore, it develops a targeted maintenance plan for the building and realizes intelligent O\&M services to ensure that it is in healthy condition. This framework can greatly improve the informatization level of the construction operation and maintenance process, solve various defects in traditional O\&M, and reduce costs.

\section{The Process of Establishing the Digital Twin Operation and Maintenance Model}

The digital twin O\&M model should adopt multiple aspects, including geometric, physical, behavioral, and institutional models [32]. The basic work of establishing a virtual model is adjusting the geometric model, which realizes the visual display of geometric information such as the size, shape, and position of the physical entity. This is consistent with the characteristics of BIM technology, which is well suited for the implementation of this step and can construct geometric shapes. Based on the BIM model, combined with IoT and intelligent sensor technology, the physical information will be integrated with the geometric model to establish the physical model. On this basis, the behavior model develops the ability to self-evolve by analyzing the relevant supplemented data. The related data are collected by computer maintenance management systems (CMMS) and building automation systems (BAS). Further, the behavior model is established by combining the changing characteristics of the physical model, such as the changing rules of material properties and the dynamic properties of structural components [33,34]. Finally, a rule model is established by combining the related norms and machine learning algorithms. The rule model can set thresholds for the O\&M data. By comparing the collected data and the thresholds, the rule model can identify the condition of the building. According to the information on the building condition, the rule model can provide methods to maintain different conditions. The specific process is shown in Figure 2.

According to the description of the digital twin O\&M model, the stages of establishing the corresponding twin may be different and it is introduced from three aspects: structure, equipment, and energy consumption.

In terms of building structure, the accumulation, processing, and utilization of building structure data can be effectively managed according to the digital model procedure. The model-based database system is connected with the relevant 3D digital model, and the corresponding digital twin building structure operation and maintenance model is established. According to the requirements of inspection practice, a three-dimensional digital model can be established by using BIM technology. The database consists of the properties of each widget and the code number of the committed file. During the operation of the building, the data generated will accumulate in the database. The models and databases are connected through a defined coding system [35]. Coding systems can often perform sophisticated data management to predict the performance of a building's overall structure throughout its life cycle. The database mainly contains the twin model data of the three dimensions of the building structure, and the three-dimensional digital model is the visual representation of the geometric data. 


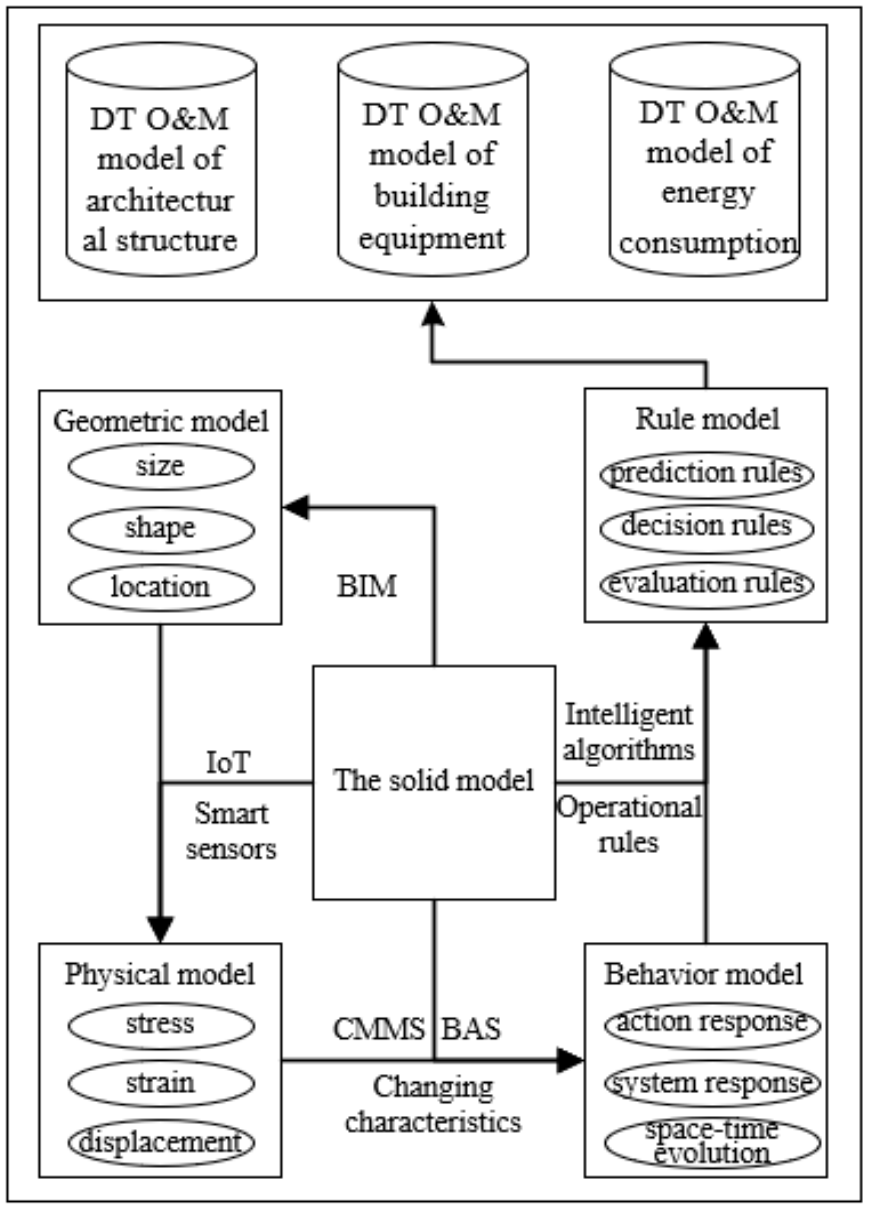

Figure 2. Flowchart of establishing a digital twin operation and maintenance model.

As for construction equipment, the system structure of BIM as the basis of the building O\&M management system forms the underlying data by combining the BIM model and equipment parameter data together with the data from the building equipment O\&M to generate the digital twin. Based on the digital twin system, the health of each component is analyzed and evaluated by the method of sensor acquisition and data optimization, and the function of fault prediction and healthy management is established. By realizing real-time synchronous transmission and interaction between the physical and virtual equipment, the model can accurately predict faults and manage health so that the health management mode of physical components can enter a new stage, which can be a significant advantage in perceiving faults and locating them accurately and quickly. At the same time, a corresponding maintenance plan can be made according to the operation conditions to improve the maintenance mode. Under the guidance of the digital twin system, the physical building equipment can communicate and transmit data in real time with the virtual twin device. The virtual digital equipment and physical building equipment under the guidance of the digital twins run simultaneously to analyze and evaluate the physical building, detect faults, and verify repairs in order to work out a complete repair and maintenance plan and ensure that the physical building is healthy and operating stably.

In terms of a building's energy consumption, fragmented and scattered data are combined and collected in the BIM model after the building is completed. These data will be organized and analyzed to show the O\&M phase for various energy-consuming equipment parameters. BIM model integration and further inclusion of its O\&M functions completes the digital twin model. On this basis, the energy consumption information of the sensors and equipment is collected through the digital twin, which reflects and simulates the physical building's operational elements and behaviors on the virtual management 
platform. Furthermore, real-time adjustment and iterative optimization of building energy consumption are realized.

\section{A Method of Predicting the State of Operations Driven by the Fusion of Digital Twin and Machine Learning}

\subsection{Fusion Mechanism of Digital Twins and Machine Learning}

The essence of the operation-dimensional state prediction method driven by the combination of digital twinning and machine learning is the use of digital twin technology to realize the digitization and visualization of the critical elements of building operation and maintenance in terms of geometry, physics, behavior, rules, and other aspects, and the intelligent analysis and prediction of the operational state of building O\&M through machine learning algorithms, including the building state prediction, residual life prediction, disease analysis, risk analysis, and so on. [36].

The fusion mechanism of the digital twin and machine learning is the core of the O\&M state prediction. Its primary function is to collect, process, and analyze data, and then output diagnostic and predictive results to support decision-making of related work. Among them, the digital twin layer has the function of perceptual data transmission. In building operation and maintenance, the relevant data are collected and processed by digital twins and then analyzed by layered machine learning algorithms. The digital twin processes the predictive information analyzed by the machine learning algorithm to make intelligent decisions that are transmitted to physical entities. This process is a closed loop that achieves accurate predictions of the operational status of buildings.

In this method, the machine learning algorithm is the core of data analysis and processing. In this study, the ANN algorithm is used to train the model with the data integrated into the digital twin as the data source, and the building O\&M status prediction mechanism based on the ANN is established. The prediction results are imported into the digital twin for simulation, which provides intelligent decision support for building operation and maintenance processes.

With the fusion of digital twins and machine learning, the operation state prediction method has the following characteristics: It provides real-time perceptual feedback based on digital twins; the digital twin model of the building O\&M process is established by collecting relevant information about the physical entity; and this model contains the whole unit with multidimensional and multiscale information of the building, which is a synchronous model realizing the digitization of the operation and maintenance process.

By combining digital twins with machine learning algorithms, this study made full use of various data sources-for example, basic building data and real-time data collected by sensors. The emergence of building O\&M problems can be effectively avoided by using machine learning for the analysis of various data and the intelligent diagnosis and prediction of the building running status, and then receiving feedback to the building entities through the twin models.

\subsection{Operation-Based State Prediction Mechanism Based on Neural Network}

In the process of operation and maintenance, the health of a building will be disturbed by many influencing factors. Different factors need to be considered for the operation and maintenance of the different building structures or construction equipment operation, and these are affected by other key factors. Therefore, this paper introduces an algorithm to predict the subsequent conditions of these key influencing factors. The predicted results are fed back to the control system.

Most building changes happen during operation. It is difficult for nonlinear processes and traditional mathematical models to obtain ideal prediction results. As a commonly used mathematical modeling method, an artificial neural network can adapt to large samples. Based on the study of historical data, it can discover the mapping relationship between the input and output, then extract and store the mapping during the learning process. In the prediction stage, according to the input of the network, we can get the next period forecast 
output, and, with machine learning data from a large number of samples, reduce the error of state assessment so as to get the correct result when dealing with these uncertainties. At the same time, the artificial neural network can store and organize data and has good generalization ability. Therefore, in a production environment where large amounts of data have been collected, artificial neural networks can be used as an important technical means of constructing a digital twin prediction system [37,38].

The neural network goes through two steps, feed-forward and reverse propagation [39]. The logical sigmoid activation function is used in both hidden and output layers because it can guide the training process. The logical sigmoid activation function is shown below. The structure and mathematical representation of the artificial neurons are shown in Figure 3.

$$
\begin{aligned}
& f(\text { sum })=\frac{1}{1+\mathrm{e}^{- \text {sum }}} \\
& \text { sum }=\sum_{i=1}^{n} X_{i} W_{i}+B
\end{aligned}
$$

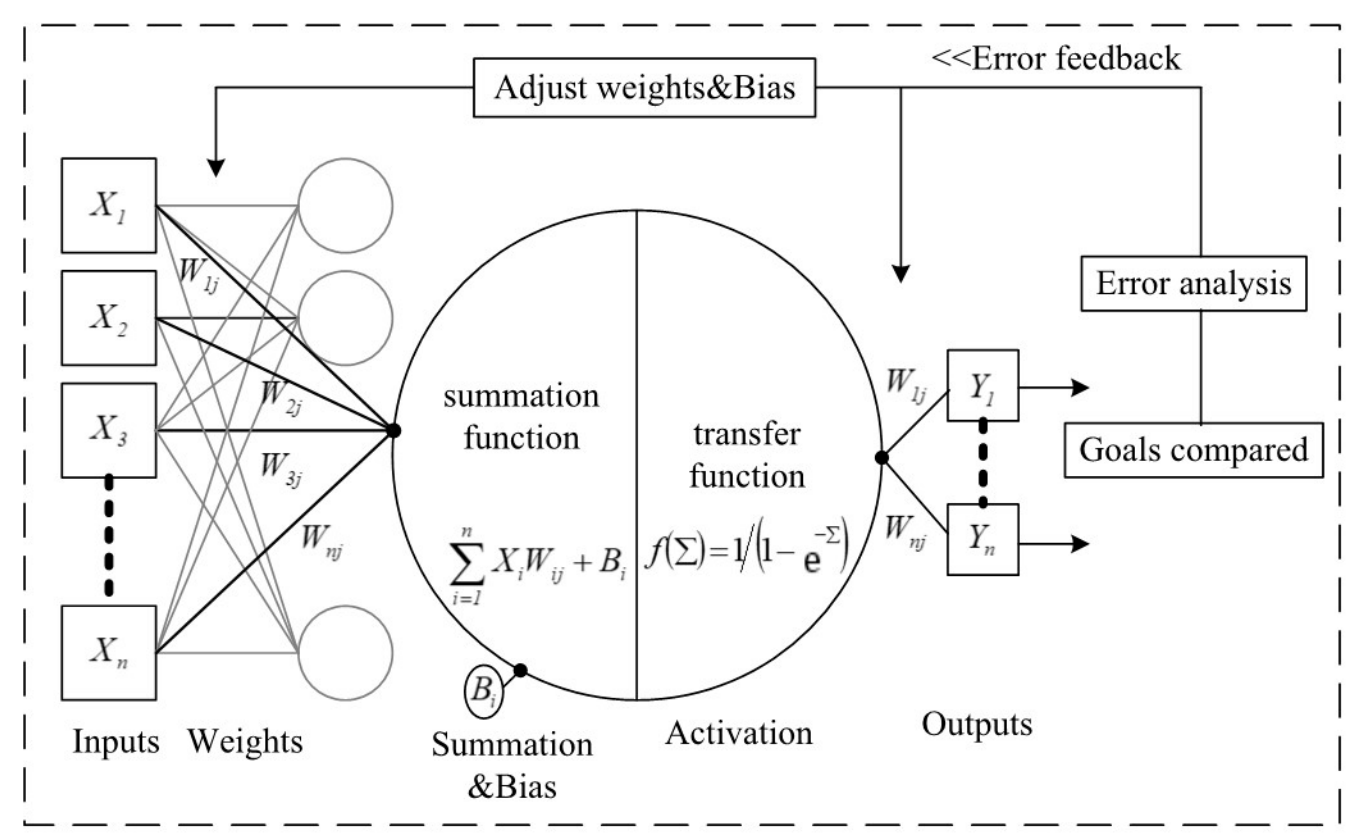

Figure 3. Structure and mathematical expression of artificial (hidden) neuron.

Here, $f($ sum $)$ is an activation function, sum is the weighted sum, $X_{i}$ is the input of neuron $i, W_{i}$ is the connection weight between two neurons, and $B$ is the deviation value. Back-propagation (BP) is one of many algorithms used to train ANNs. The algorithm continuously propagates the error backward and adjusts each network weight and deviation value (output and hidden layer) to minimize the direction of the network output error. This makes the predicted output closer to the target output, thus reducing the error of the entire network to a satisfactory level. Many techniques can be used in reverse propagation algorithms, such as gradient descent, Newtonian, and Levenberg-Marquardt (LM). LM is mainly used in this study.

\subsection{Evaluation of Predictive Models}

In order to verify the applicability of the ANN, it is built to predict the cable force of a spoke-type steel frame. The debugging process refers to the simulation analysis done by Liu [40]. In this test, $70 \%$ is training data, $15 \%$ is validation data, and $15 \%$ is test data, as obtained by Ansys. The input is the displacement of 30 nodes in the model and the number of neurons is 30 . Due to space limitations, only partial data are included, as shown in Table 1. The output layer parameter is the cable force of the lower ring. 
Table 1. Input data of ANN.

\begin{tabular}{|c|c|c|c|c|c|c|c|c|c|c|}
\hline Node & Case1 & Case2 & Case3 & Case4 & Case5 & Case6 & Case7 & Case8 & Case9 & Case10 \\
\hline node1 & 0.0 & 0.0 & 0.0 & 0.0 & 0.0 & 0.0 & 0.0 & 0.0 & 0.0 & 0.0 \\
\hline node2 & 0.0 & 0.1 & 0.2 & 0.3 & 0.4 & 0.5 & 0.6 & 0.7 & 0.8 & 0.9 \\
\hline node3 & 0.0 & 0.1 & 0.2 & 0.3 & 0.4 & 0.5 & 0.6 & 0.7 & 0.8 & 0.9 \\
\hline node4 & 0.0 & 0.1 & 0.2 & 0.3 & 0.4 & 0.5 & 0.6 & 0.7 & 0.8 & 0.9 \\
\hline node5 & 0.0 & 0.1 & 0.2 & 0.3 & 0.4 & 0.5 & 0.6 & 0.7 & 0.8 & 0.9 \\
\hline node6 & 0.0 & 0.1 & 0.2 & 0.3 & 0.4 & 0.5 & 0.6 & 0.7 & 0.8 & 0.9 \\
\hline node7 & 0.0 & 0.1 & 0.2 & 0.3 & 0.4 & 0.5 & 0.6 & 0.7 & 0.8 & 0.9 \\
\hline node8 & 0.0 & 0.1 & 0.2 & 0.3 & 0.4 & 0.5 & 0.6 & 0.7 & 0.8 & 0.9 \\
\hline node9 & 0.0 & 0.1 & 0.2 & 0.3 & 0.4 & 0.5 & 0.6 & 0.7 & 0.8 & 0.9 \\
\hline node10 & 0.0 & 0.1 & 0.2 & 0.3 & 0.4 & 0.5 & 0.6 & 0.7 & 0.8 & 0.9 \\
\hline node11 & 0.0 & 0.1 & 0.2 & 0.3 & 0.4 & 0.5 & 0.6 & 0.7 & 0.8 & 0.9 \\
\hline node12 & 0.0 & 0.1 & 0.2 & 0.3 & 0.4 & 0.5 & 0.6 & 0.7 & 0.8 & 0.9 \\
\hline node13 & 0.0 & 0.1 & 0.2 & 0.3 & 0.4 & 0.5 & 0.6 & 0.7 & 0.8 & 0.9 \\
\hline node14 & 0.0 & 0.1 & 0.2 & 0.3 & 0.4 & 0.5 & 0.6 & 0.7 & 0.8 & 0.9 \\
\hline node15 & 0.0 & 0.1 & 0.2 & 0.3 & 0.4 & 0.5 & 0.6 & 0.7 & 0.8 & 0.9 \\
\hline node16 & 0.0 & 0.1 & 0.2 & 0.3 & 0.4 & 0.5 & 0.6 & 0.7 & 0.8 & 0.9 \\
\hline node17 & 0.0 & 0.1 & 0.2 & 0.3 & 0.4 & 0.5 & 0.6 & 0.7 & 0.8 & 0.9 \\
\hline node18 & 0.0 & 0.1 & 0.2 & 0.3 & 0.4 & 0.5 & 0.6 & 0.7 & 0.8 & 0.9 \\
\hline node19 & 0.0 & 0.1 & 0.2 & 0.3 & 0.4 & 0.5 & 0.6 & 0.7 & 0.8 & 0.9 \\
\hline node20 & 0.0 & 0.1 & 0.2 & 0.3 & 0.4 & 0.5 & 0.6 & 0.7 & 0.8 & 0.9 \\
\hline node21 & 0.0 & 0.1 & 0.2 & 0.3 & 0.4 & 0.5 & 0.6 & 0.7 & 0.8 & 0.9 \\
\hline node22 & 0.0 & 0.1 & 0.2 & 0.3 & 0.4 & 0.5 & 0.6 & 0.7 & 0.8 & 0.9 \\
\hline node23 & 0.0 & 0.1 & 0.2 & 0.3 & 0.4 & 0.5 & 0.6 & 0.7 & 0.8 & 0.9 \\
\hline node24 & 0.0 & 0.1 & 0.2 & 0.3 & 0.4 & 0.5 & 0.6 & 0.7 & 0.8 & 0.9 \\
\hline node25 & 0.0 & 0.1 & 0.2 & 0.3 & 0.4 & 0.5 & 0.6 & 0.7 & 0.8 & 0.9 \\
\hline node26 & 0.0 & 0.1 & 0.2 & 0.3 & 0.4 & 0.5 & 0.6 & 0.7 & 0.8 & 0.9 \\
\hline node27 & 0.0 & 0.1 & 0.2 & 0.3 & 0.4 & 0.5 & 0.6 & 0.7 & 0.8 & 0.9 \\
\hline node28 & 0.0 & 0.1 & 0.2 & 0.3 & 0.4 & 0.5 & 0.6 & 0.7 & 0.8 & 0.9 \\
\hline node29 & 0.0 & 0.1 & 0.2 & 0.3 & 0.4 & 0.5 & 0.6 & 0.7 & 0.8 & 0.9 \\
\hline node30 & 0.0 & 0.1 & 0.2 & 0.3 & 0.4 & 0.5 & 0.6 & 0.7 & 0.8 & 0.9 \\
\hline
\end{tabular}

In this study, a hidden layer was used and the number of input and output parameters for building operation and maintenance were known. In order to find the optimal structure of the neural network, only the number of implied neurons needs to be calculated. In general, too many implicit layer neurons will make the training too long, and overtraining will lead to overfitting problems. There are too few implicit neurons to predict the data adequately. It is calculated according to the empirical formula:

$$
n=\sqrt{m+l}+a
$$

where $n$ is the number of implicit layer neurons, $l$ is the number of input layer cells, $m$ is the number of output layer nodes, and $a$ is the adjustment constant between 1 and 10 .

This section studies the influence of different numbers of neurons in the hidden layer on the accuracy of the ANN model. A variable structure method that gradually increases the number of hidden layer units was adopted based on Empirical Formula (4). That is, relatively few hidden layer cells are added at the beginning. After learning a certain number of times, the ideal number of hidden layer cells is finally reached by increasing the number of hidden layer cells for debugging. Based on the training data, when the number of hidden layer cells is $10, R^{2}$ met the requirements and did not take much training time. It was determined to be 10 . The network allows the maximum error to be set at 0.001 and the learning rate at 0.01 for network training. The developed model was then statistically evaluated by using mean square error (MSE), coefficient of determination $\left(R^{2}\right)$, and mean absolute error (MAE), which are defined in Equations (5)-(7) below. In short, the lower the value, the better the network performance. $R$ measures the correlation between the 
output and target in the network; $R=1$ indicates a close relationship and $R=0$ represents a random association.

$$
\begin{gathered}
M S E=\frac{1}{n} \sum_{i=1}^{n}\left(T_{i}-O_{i}\right)^{2} \\
R^{2}=1-\left(\frac{\sum_{i=1}^{n}\left(T_{i}-O_{i}\right)^{2}}{\sum_{i=1}^{n}\left(O_{i}\right)^{2}}\right) \\
M A E=\frac{1}{N} \sum_{i=1}^{N}\left|X_{i}-Y_{i}\right|
\end{gathered}
$$

Here, $n$ is the total number of training datasets, and $T_{i}$ and $O_{i}$ are the target (training sample) and ANN output value, respectively.

The mean error in the LM network training, validation, and testing phases decreases rapidly with increased iterations. Since the test set and validation set error have similar characteristics, the MSE results are acceptable. As is shown in Table 2, for the training and testing input data for MSE, MAE statistics are $7.62 \times 10^{-5}, 0.0297$ and $8.53 \times 10^{-5}, 0.0366$. The artificial neural network model indicates an excellent correlation between the predicted and measured values. In addition, a higher $\mathrm{R}^{2}$ value and lower MSE value indicate that the ANN model has better prediction ability and generalization performance.

Table 2. Performance of neural network model.

\begin{tabular}{cccc}
\hline & MSE & $\boldsymbol{R}^{\mathbf{2}}$ & MAE \\
\hline Training & $7.62 \times 10^{-5}$ & 0.994 & 0.0297 \\
Validation & $9.81 \times 10^{-5}$ & 0.992 & 0.0334 \\
Test & $8.53 \times 10^{-5}$ & 0.995 & 0.0366 \\
All data & $8.09 \times 10^{-5}$ & 0.994 & 0.0313 \\
\hline
\end{tabular}

4.4. Building Operations State Prediction Process Based on Digital Twins and Machine Learning Algorithms

According to the modeling method described in this paper, the digital twin model of the construction operation and maintenance stage can be established, and the corresponding parameters in the virtual space can be updated in real-time according to the entity monitoring data in order to map the real-time state of the building in the physical space to the virtual space and realize a real-time visualization of the construction operation and maintenance. Using an artificial neural network algorithm, we can calculate the operation and maintenance data of the next period with the data processing of entity monitoring and virtual model-related information. The results are fed back to the virtual model to realize real-time prediction and visualization.

The specific prediction process is shown in Figure 4. The data of moment $\mathrm{t}$ are the real-time data and the data of moment $t+1$ are the forecast data. The key factors of moment $t$ in the model are entered into the algorithm layer with ANN as the core. The key influencing factors of moment $t+1$ are deduced and fed back into the model to obtain the decisive elements of moment $t+1$ of the construction operation and maintenance state, and to determine the operation and maintenance status of the building at time $t+1$. Finally, it determines whether there is a problem with the building and if maintenance is required.

BIM, building automation system (BAS), and IoT echo the digital twin operation and maintenance mode in the establishment process above. They represent the three main data sources of the digital twin model. BIM technology represents basic data, such as building geometry information contained in the BIM model. BAS represents the relevant operational data obtained for monitoring various facilities and equipment in the building. IoT technology represents building operations data collected through intelligent sensors with IoT technology at the core. 


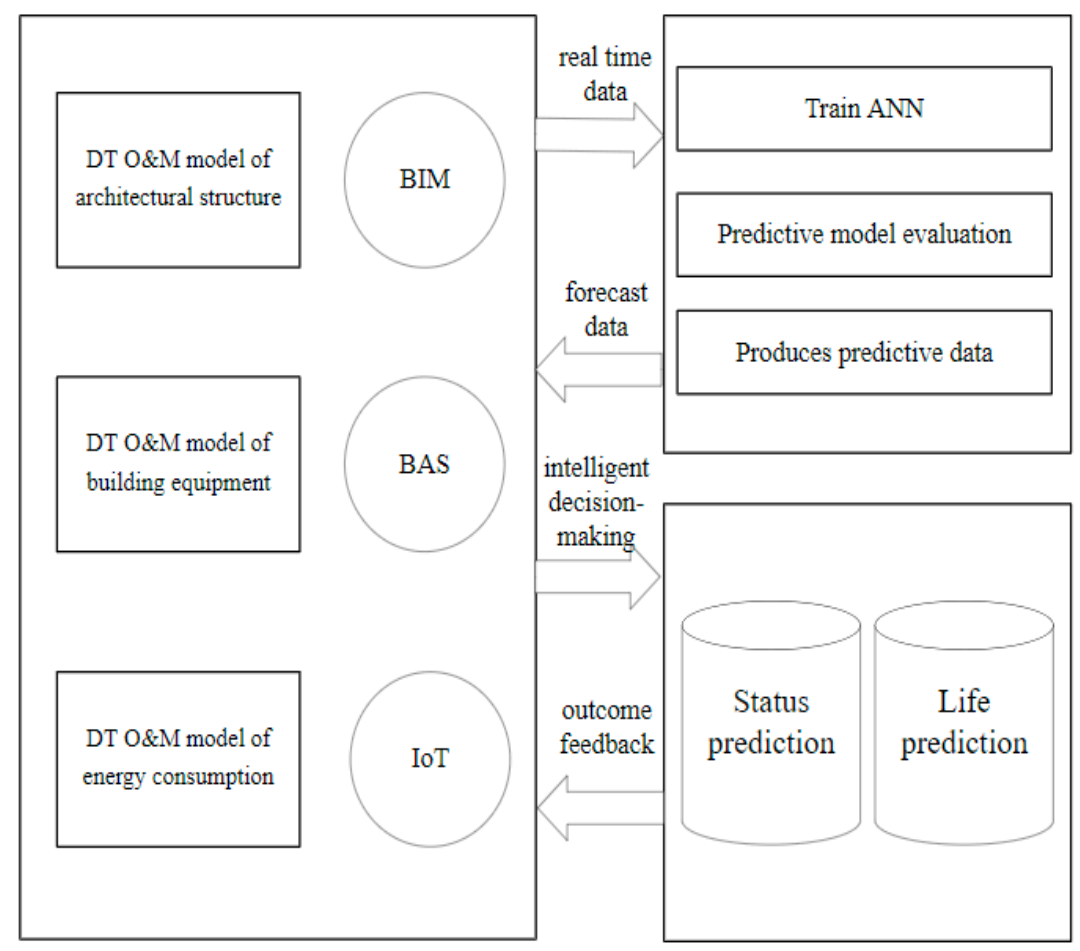

Figure 4. Operational and maintenance prediction process.

\subsection{Analysis of the Prediction Process}

This study uses the experiment of Liu as a reference [41]. The test model is shown in Figure 5. The prediction model is used to explore 47 different working conditions of pre-stress loss scenarios. To simulate the relaxation limit state of the cable, the percentage of pre-stress applied in the finite element simulation was $0-100 \%$. The experiment analyzed a single upper radial cable, a single lower radial cable, an upper ring cable, a lower ring cable, a total upper radial cable, and a total lower radial cable. Through finite element simulation, it can be seen that the relaxation of a single upper or lower radial cable has little effect on the static performance of the structure. Therefore, the single upper and lower radial cables are subjected to $50 \%$ and $100 \%$ relaxation of pre-stress applied in the simulation. The rest is relaxed according to $20,40,60,80$, and $100 \%$ of the pre-stress applied. Some of the working conditions are shown in Table 3. The input layer includes 12 input neurons, 5 in the lower radial cable, 5 in the upper radial cable, and 2 in the residual relaxation pre-stress of the upper and lower cables. The hidden layer contains 10 neurons and the output neuron is the displacement of the maximum node of the inner strut. The input data are fed to the digital twin model by IoT and sensors.

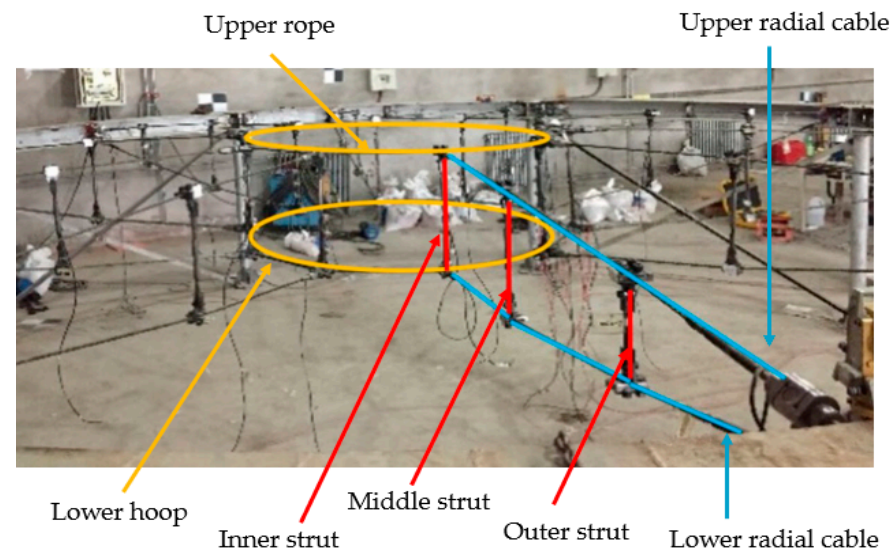

Figure 5. Test model. 
Table 3. Percentage of slack residual pre-stress of cable members.

\begin{tabular}{ccccccccccccc}
\hline & \multicolumn{4}{c}{ Uase } & \multicolumn{4}{c}{ Upper Radial Cable } & \multicolumn{9}{c}{ Lower Radial Cable } & Upper & $\begin{array}{c}\text { Lower } \\
\text { Rope }\end{array}$ \\
\hline & $\mathbf{1}$ & $\mathbf{2}$ & $\mathbf{3}$ & $\mathbf{4}$ & $\mathbf{5}$ & $\mathbf{6}$ & $\mathbf{7}$ & $\mathbf{8}$ & $\mathbf{9}$ & $\mathbf{1 0}$ & & \\
\hline 1 & 0.6 & 0.6 & 0.6 & 0.6 & 0.6 & 1 & 1 & 1 & 1 & 1 & 1 & 1 \\
2 & 0.4 & 0.4 & 0.4 & 0.4 & 0.4 & 1 & 1 & 1 & 1 & 1 & 1 & 1 \\
3 & 0.2 & 0.2 & 0.2 & 0.2 & 0.2 & 1 & 1 & 1 & 1 & 1 & 1 & 1 \\
4 & 0 & 0 & 0 & 0 & 0 & 1 & 1 & 1 & 1 & 1 & 1 & 1 \\
5 & 1 & 1 & 1 & 1 & 1 & 0.8 & 0.8 & 0.8 & 0.8 & 0.8 & 1 & 1 \\
6 & 1 & 1 & 1 & 1 & 1 & 0.6 & 0.6 & 0.6 & 0.6 & 0.6 & 1 & 1 \\
7 & 1 & 1 & 1 & 1 & 1 & 0.4 & 0.4 & 0.4 & 0.4 & 0.4 & 1 & 1 \\
8 & 1 & 1 & 1 & 1 & 1 & 0.2 & 0.2 & 0.2 & 0.2 & 0.2 & 1 & 1 \\
9 & 1 & 1 & 1 & 1 & 1 & 0 & 0 & 0 & 0 & 0 & 1 & 1 \\
10 & 0.8 & 0.8 & 0.8 & 0.8 & 0.8 & 0.8 & 0.8 & 0.8 & 0.8 & 0.8 & 0.8 & 0.8 \\
11 & 0.6 & 0.6 & 0.6 & 0.6 & 0.6 & 0.6 & 0.6 & 0.6 & 0.6 & 0.6 & 0.6 & 0.6 \\
12 & 0.4 & 0.4 & 0.4 & 0.4 & 0.4 & 0.4 & 0.4 & 0.4 & 0.4 & 0.4 & 0.4 & 0.4 \\
13 & 0.2 & 0.2 & 0.2 & 0.2 & 0.2 & 0.2 & 0.2 & 0.2 & 0.2 & 0.2 & 0.2 & 0.2 \\
14 & 0 & 0 & 0 & 0 & 0 & 0 & 0 & 0 & 0 & 0 & 0 & 0 \\
15 & 1.2 & 1.2 & 1.2 & 1.2 & 1.2 & 1.2 & 1.2 & 1.2 & 1.2 & 1.2 & 1.2 & 1.2 \\
16 & 1.4 & 1.4 & 1.4 & 1.4 & 1.4 & 1.4 & 1.4 & 1.4 & 1.4 & 1.4 & 1.4 & 1.4 \\
\hline
\end{tabular}

According to the established ANN model, the predicted value of the maximum displacement of the node under the inner pole under different working conditions and the relative error value are obtained. As shown in Figure 6, there is not much difference between the actual data from the experiment and the predicted data of this model. The determination coefficient $R^{2}$ reached 0.9866 , and the process took just a few seconds. Given this, it is an efficient and accurate forecasting process. just a few seconds. So, it is an efficient and accurate forecasting process.

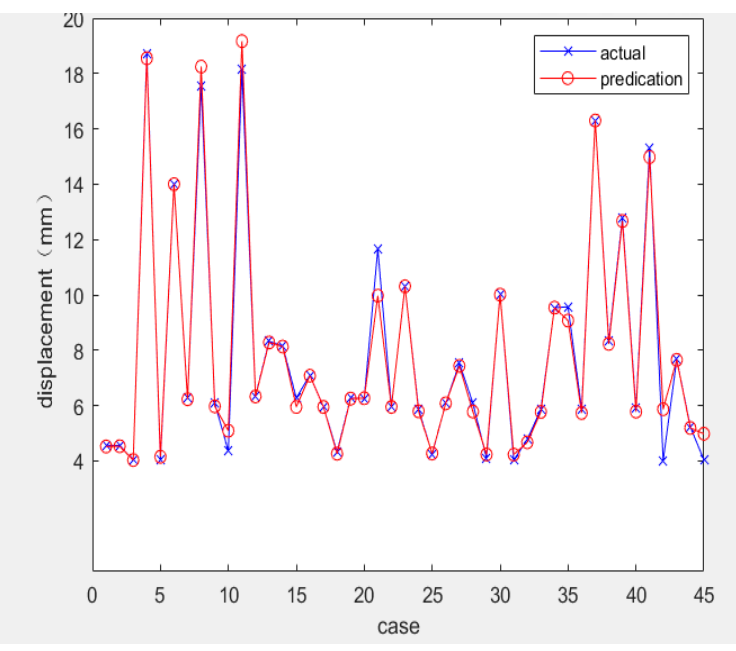

(a)

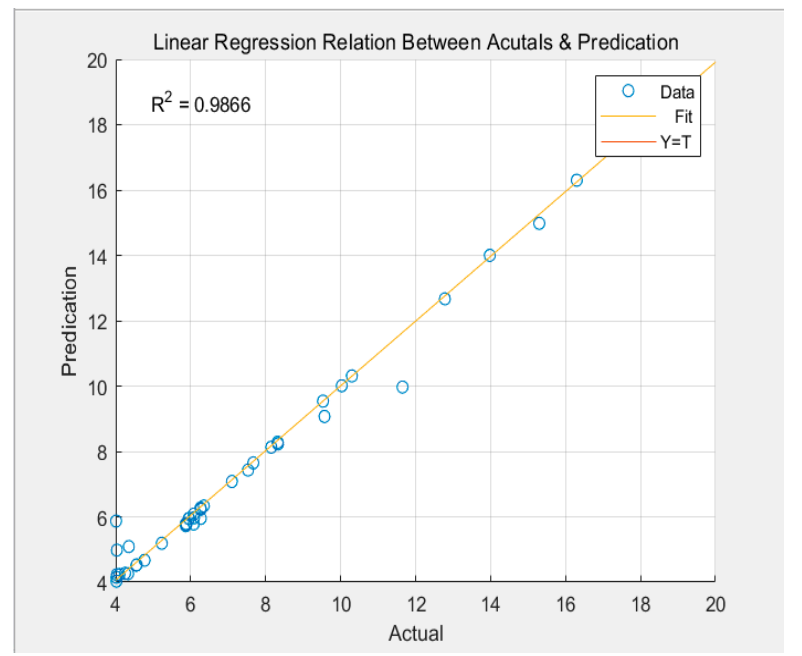

(b)

Figure 6. (a) Comparison and (b) correlation between actual and predicted data.

\section{Development Process of the Operation and Maintenance System Based on Digital Twin Technology}

5.1. Development Route Based on Digital Twin Technology Operation and Maintenance Platform Function

According to the process for the development of the digital twin technology operation and maintenance platform, the model mentioned above establishes the process and state prediction method as the core and determines the specific functional development route of the platform. First of all, the BIM model is lightweight, and the dynamic information collected by the operation stage through the Internet of Things is integrated into it to form 
the digital twin model. Then, through the network platform technology and machine learning algorithm, the operation and maintenance management platform based on digital twin technology is created. The main functions of the platform include scene roaming of operational targets and data visualization management. Scene roaming includes overall and local roaming; data visualization management includes information on the O\&M of the target building environment, energy consumption, components, component mechanics, equipment, and space use. Through data analysis, the platform's functions of emergency warning, energy consumption early warning, and maintenance early warning are realized.

A specific development flowchart for the digital twin platform functionality is shown in Figure 7.

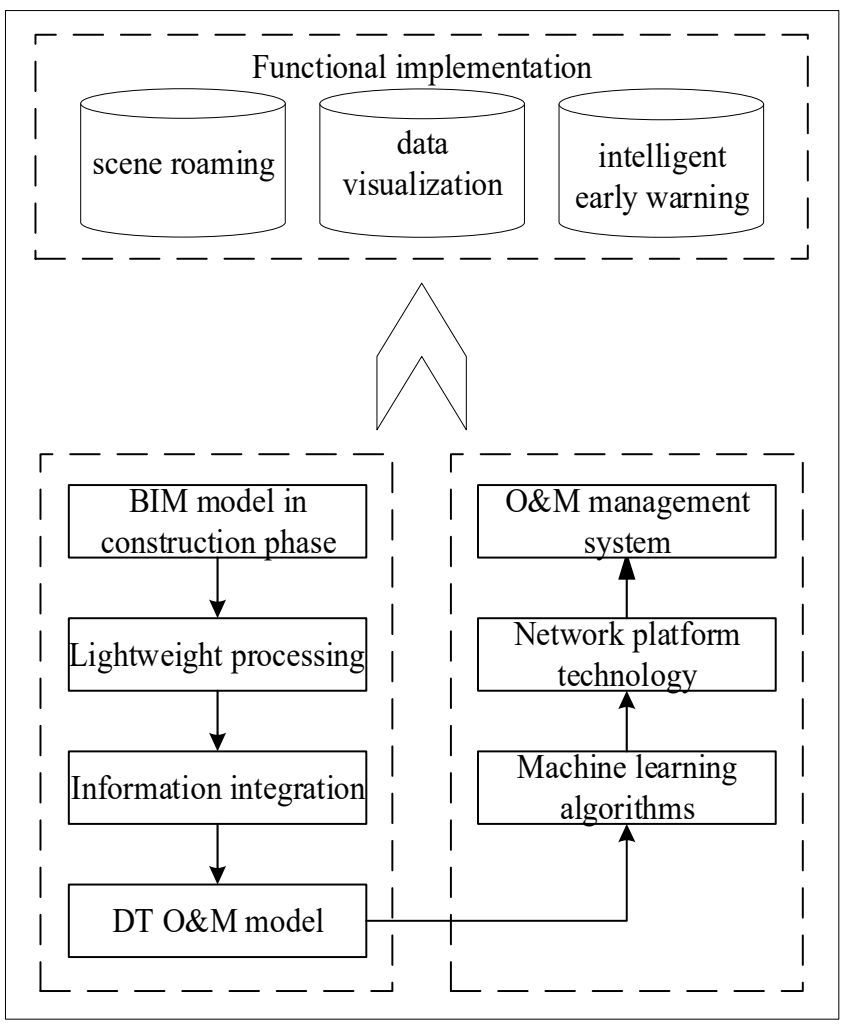

Figure 7. Flowchart of development route based on digital twin technology operation and maintenance platform function.

\subsection{Overall Architecture of the Operating System Development Based on Digital Twin Technology}

Establishing an operation and maintenance system should start with realizing the three major objectives of efficient operation and maintenance according to the functional, platform, data, network, and physical layers in reverse order to design the details of each layer, thereby completing the platform construction. Platform construction follows the function, perception, virtual model, transmission, and data layers to achieve forward data transmission. Finally, the platform layer is aimed at achieving green, efficient, and safe operation and maintenance as the three major objectives. Figure 8 shows the overall architecture of the digital twin platform. The system architecture contains the following layers:

Platform functional layer: The platform functional layer includes all kinds of analytical decision-making systems. In practical applications, production management personnel make the digital twin calculations and analyze the data and results as the basis for analysis and decision-making. The formation of information and decision-making becomes a closed loop. This layer is mainly responsible for interacting with end users by granting different user rights within their prescribed permissions. Administrators can log in for data entry, query, modification, and other operations. This layer is implemented primarily on the Web. For example, sensors detect changes in stress on a layer of the beam of a monitoring target 
and record that if the stress changes significantly beyond the safe value range, then the structural inspection system accessed by the platform alerts the management that structural reinforcement or maintenance is required. After logging in to the platform, management personnel can find problems and report them to the corresponding personnel to solve them through the mobile terminal.

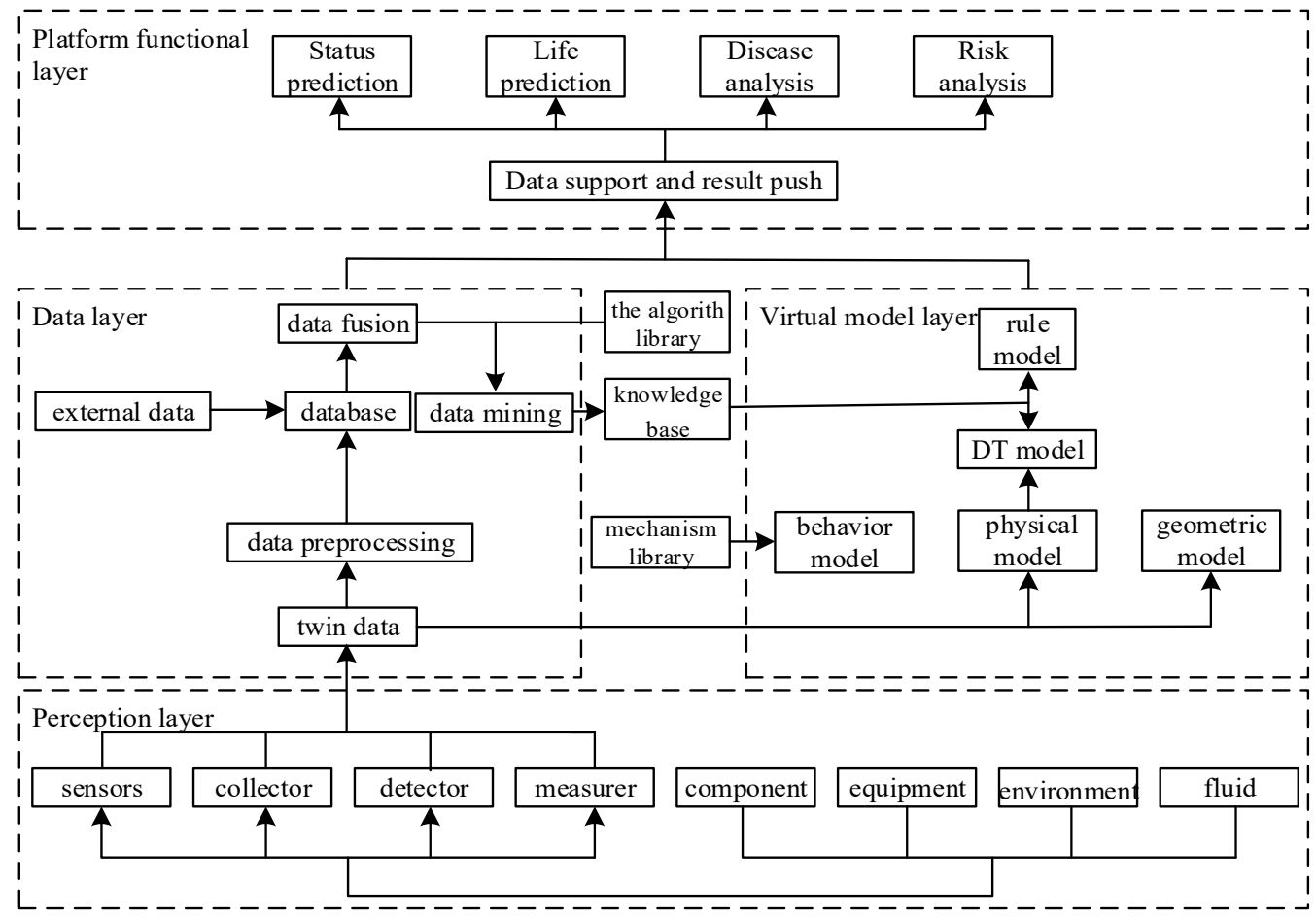

Figure 8. Overall architecture of operation and maintenance platform based on digital twin technology.

Platform functional layer: The platform functional layer includes all kinds of analytical decision-making systems. In practical applications, production management personnel make the digital twin calculations and analyze the data and results as the basis for analysis and decision-making. The formation of information and decision-making becomes a closed loop. This layer is mainly responsible for interacting with end users by granting different user rights within their prescribed permissions. Administrators can log in for data entry, query, modification, and other operations. This layer is implemented primarily on the Web. For example, sensors detect changes in stress on a layer of the beam of a monitoring target and record that if the stress changes significantly beyond the safe value range, then the structural inspection system accessed by the platform alerts the management that structural reinforcement or maintenance is required. After logging in to the platform, management personnel can find problems and report them to the corresponding personnel to solve them through the mobile terminal.

Perception layer: The perception layer contains various physical entities in the actual building system and supports the detection devices and other content. The entity side collects the information, operating parameters, and operation instructions of the actual construction system and passes them, accurately, to the data measurement. The perception layer records the data description of the entire model and describes the basic information that is needed for each professional application. This layer contains information about the surrounding environment of the building, including the information recorded by sensors such as cameras, as well as temperature, humidity, smoke, etc. This layer also contains general information about the building entity, such as the information recorded by stress sensors, strain sensors, and so on. The perceptive layer also detects equipment information such as water volume, electricity, and so on. 
Virtual model layer: The virtual model layer is mainly based on the simulation and rule models. The simulation model covers the rule model, which reflects the objective law of the actual construction system; the physical model, which embodies the information of the system components such as attributes, features, and so on, and the geometry model, which includes the shape, size, position, and assembly relationship of the key components of the construction system. The rule model supports the construction and calculation of the simulation model through the results obtained from experience or data analysis.

Transport layer: The most important function of the transport layer is to convert the collected data to the upper layer. Data are collected from physical devices connected to physical assets/spaces in the building or its surroundings. This layer can use a variety of communication technologies, such as 5G or low-power, wide-area networks (LPs-WANs). Among the available technologies, wireless local area network (WLAN) technology (i.e., wireless networks) is one of the most well-known and widely used.

Data layer: The data layer is responsible for providing data read and write support for the virtual model layer, which collects real-time state data from the perceptive layer to enable the multi-source heterogeneous data stores, which convert data from different systems, formats, and manufacturers into exposed standardized data formats and store it in a specified database. The data source stored in the database is divided into two parts, twin data and external data. Twin data refers to the building's digital twin operation and maintenance model, which contains all kinds of data. External data mainly include operational experience information and knowledge of other buildings for reference. The database of continuous dynamic updates of synchronous building operations mainly provides the centralized storage of information, rapid retrieval updates, and data management. All operations related to the consistent integrity of data security are carried out in the data layer.

\section{Discussion}

Because of its real-time interaction and evolution, digital twin technology is suitable for and a key technology in building operation and maintenance processes. According to this conception, this paper constructs a digital twin model for these processes. Machine learning algorithms provide analysis for massive amounts of complex data generated during operations [42]. The results from this study support the argument by verifying the ANN. The reliability of the output result is determined by checking the MSE, $R^{2}$, and MAE values. When the ANN is used to predict the cable force of the lower ring, the values meet the requirement, as shown in Table 2.

This paper proves that the fusion of digital twins and machine learning is feasible in Section 4.5. As shown in Figure 6, the error between the actual and predicted values is basically less than $1 \mathrm{~mm}$, and the determination coefficient $R^{2}$ is approximately 0.98 . The mechanism is reliable and efficient.

This paper puts forward a method to develop an O\&M platform based on digital twins, but it stays at the theoretical level. Its feasibility is supported by the prediction method's verification in Section 4. The study of the practical application of this method still needs much work.

\section{Conclusions}

This paper introduces the idea of digital twin technology for the process of building operation and maintenance, as well as the fusion mechanism of digital twin technology and a machine learning algorithm, to realize the intelligent prediction of building operation and maintenance status.

1. According to the characteristics of virtual interaction and real-time feedback of digital twin technology, a digital twin framework oriented to building operation and maintenance is proposed, which provides a new idea for the practical application of digital twins in the process of intelligent building operation and maintenance.In the process of combining digital twin technology with a machine learning algorithm, this paper 
first puts forward the process of establishing a digital twin operation and maintenance model to realize the integration and visual display of building operation and maintenance data. The machine learning algorithm is then applied for data-driven intelligent prediction and diagnosis of the building operation and maintenance processes.

2. The operation and maintenance processes of a building produce large amounts of diverse data. With digital twin technology as the core, the data are visual and can be retrieved from a 3D model, which is clearer than the traditional way.

3. Based on digital twin technology and the machine learning algorithm, the development of an operation and maintenance system is proposed, providing the idea for the application of digital twin technology in intelligent operation and maintenance.

In summary, the application of the digital twin technology and machine learning algorithm is an effective way to achieve intelligent prediction and diagnosis of building operation and maintenance status in order to achieve intelligent operation and maintenance of a building. However, the implementation of digital twin technology is still in its initial stages. There are still certain limitations regarding the establishment of digital twins. Digital twins still have much potential to be developed in O\&M. Therefore, most of the methods proposed in this paper only exist at the theoretical level; it is difficult to prove them with practical examples. We will try to use the theory to establish a platform and apply it to a practical project in future work.

Author Contributions: Conceptualization, Z.L.; methodology, Y.Z.; software, Y.Z.; validation, Y.Z., N.W., Z.L. and E.M.; writing-original draft preparation, N.W. and E.M.; writing-review and editing, Y.Z.; project administration, Z.L.; funding acquisition, Z.L. All authors have read and agreed to the published version of the manuscript.

Funding: This research was supported by the Deep Learning Based Lifting Safety Risk Prediction and Control Method of Assemblies Building (8201001) of Beijing Natural Science Foundation.

Institutional Review Board Statement: Not applicable.

Informed Consent Statement: Not applicable.

Data Availability Statement: The data used to support the findings of this study are available from the corresponding author upon request.

Acknowledgments: The authors would like to thank the Beijing University of Technology for their support throughout the research project.

Conflicts of Interest: The authors declare no conflict of interest.

\section{References}

1. Edirisinghe, R.; Setunge, S.; Zhang, G. Markov Model—Based Building Deterioration Prediction and ISO Factor Analysis for Building Management. J. Manag. Eng. 2015, 31, 04015009. [CrossRef]

2. Park, S.; Ahn, Y.; Lee, S. Analyzing the Finishing Works Service Life Pattern of Public Housing in South Korea by Probabilistic Approach. Sustainability 2018, 10, 4469. [CrossRef]

3. Park, M.; Kwon, N.; Lee, J.; Lee, S.; Ahn, Y. Probabilistic Maintenance Cost Analysis for Aged Multi-Family Housing. Sustainability 2019, 11, 1843. [CrossRef]

4. Silva, A.; de Brito, J. Do we need a buildings' inspection, diagnosis and service life prediction software? J. Build. Eng. 2019, 22, 335-348. [CrossRef]

5. Chan, D.W. Sustainable building maintenance for safer and healthier cities: Effective strategies for implementing the Mandatory Building Inspection Scheme (MBIS) in Hong Kong. J. Build. Eng. 2019, 24, 100737. [CrossRef]

6. Yang, P.; Jia, R.; Jian, P.Z.; Zhen, H.H. A hybrid data mining approach on BIM-based building operation and maintenance. Build. Environ. 2017, 126, 483-495. [CrossRef]

7. Yu, W.; Bai, J.; Li, H. Building Digital Operation and Maintenance Based on BIM. IOP Conf. Ser. Earth Environ. Sci. 2021, 634, 012044. [CrossRef]

8. Lee, S.; Akin, Ö. Augmented reality-based computational fieldwork support for equipment operations and maintenance. Autom. Constr. 2011, 20, 338-352. [CrossRef]

9. Taffese, W.; Nigussie, E.; Isoaho, J. Internet of Things based Durability Monitoring and Assessment of Reinforced Concrete Structures. Procedia Comput. Sci. 2019, 155, 672-679. [CrossRef] 
10. Yasuda, S.; Miyazaki, S. Fatigue Crack Detection System Based on IoT and Statistical Analysis. Procedia CIRP 2017, 61, 785-789. [CrossRef]

11. Ghiasi, R.; Ghasemi, M.R.; Chan, T.H.T. Optimum feature selection for SHM of benchmark structures using efficient AI mechanism. Smart Struct. Syst. 2021, 27, 623-640. [CrossRef]

12. Bernardini, G.; Di Giuseppe, E.; D'Orazio, M.; Quagliarini, E. Occupants' Behavioral Analysis for the Optimization of Building Operation and Maintenance: A Case Study to Improve the Use of Elevators in a University Building. In Sustainability in Energy and Buildings 2020; Springer: Singapore, 2021; Volume 203, pp. 207-217. [CrossRef]

13. Autiosalo, J.; Vepsalainen, J.; Viitala, R.; Tammi, K. A Feature-Based Framework for Structuring Industrial Digital Twins. IEEE Access 2019, 8, 1193-1208. [CrossRef]

14. Kritzinger, W.; Karner, M.; Traar, G.; Henjes, J.; Sihn, W. Digital Twin in manufacturing: A categorical literature review and classification. IFAC-Pap. OnLine 2018, 51, 1016-1022. [CrossRef]

15. Lee, J.; Lapira, E.; Bagheri, B.; Kao, H.-A. Recent advances and trends in predictive manufacturing systems in big data environment. Manuf. Lett. 2013, 1, 38-41. [CrossRef]

16. Talkhestani, B.A.; Jung, T.; Lindemann, B.; Sahlab, N.; Jazdi, N.; Schloegl, W.; Weyrich, M. An architecture of an Intelligent Digital Twin in a Cyber-Physical Production System. Automatisierungstechnik 2019, 67, 762-782. [CrossRef]

17. Sepasgozar, S.M.E. Differentiating Digital Twin from Digital Shadow: Elucidating a Paradigm Shift to Expedite a Smart, Sustainable Built Environment. Buildings 2021, 11, 151. [CrossRef]

18. Tao, F.; Cheng, J.; Qi, Q.; Zhang, M.; Zhang, H.; Sui, F. Digital twin-driven product design, manufacturing and service with big data. Int. J. Adv. Manuf. Technol. 2018, 94, 3563-3576. [CrossRef]

19. Warke, V.; Kumar, S.; Bongale, A.; Kotecha, K. Sustainable Development of Smart Manufacturing Driven by the Digital Twin Framework: A Statistical Analysis. Sustainability 2021, 13, 10139. [CrossRef]

20. Cai, Y.; Wang, Y.; Burnett, M. Using augmented reality to build digital twin for reconfigurable additive manufacturing system. $J$. Manuf. Syst. 2020, 56, 598-604. [CrossRef]

21. Moretti, M.; Rossi, A.; Senin, N. In-process monitoring of part geometry in fused filament fabrication using computer vision and digital twins. Addit. Manuf. 2021, 37, 101609. [CrossRef]

22. Jiang, F.; Ma, L.; Broyd, T.; Chen, K. Digital twin and its implementations in the civil engineering sector. Autom. Constr. 2021, 130, 103838. [CrossRef]

23. Leser, P.E.; Warner, J.E.; Leser, W.P.; Bomarito, G.F.; Newman, J.A.; Hochhalter, J.D. A digital twin feasibility study (Part II): Nondeterministic predictions of fatigue life using in-situ diagnostics and prognostics. Eng. Fract. Mech. 2020, 229, 106903. [CrossRef]

24. Ye, C.; Butler, L.; Calka, B.; Iangurazov, M.; Lu, Q.; Gregory, A.; Girolami, M.; Middleton, C. A Digital Twin of Bridges for Structural Health Monitoring. Structural Health Monitoring 2019: Enabling Intelligent Life-Cycle Health Management for Industry Internet of Things (IIOT). In Proceedings of the 12th International Workshop on Structural Health Monitoring, Stanford, CA, USA, 10-12 September 2019; Volume 1, pp. 1619-1626. [CrossRef]

25. Sivalingam, K.; Sepulveda, M.; Spring, M.; Davies, P. A Review and Methodology Development for Remaining Useful Life Prediction of Offshore Fixed and Floating Wind turbine Power Converter with Digital Twin Technology Perspective. In Proceedings of the 2018 2nd International Conference on Green Energy and Applications (ICGEA), Singapore, 24-26 March 2018; pp. 197-204. [CrossRef]

26. Liu, Z.; Zhang, A.; Wang, W.; Wang, J. Dynamic Fire Evacuation Guidance Method for Winter Olympic Venues Based on Digital Twin-Driven Model. J. Tongji Univ. (Nat. Sci.) 2020, 48, 962-971. (In Chinese)

27. Liu, Z.; Zhang, A.; Xing, Z.; Sun, J.; Wang, Y.; Jiang, A.; Du, X. Research on five-dimensional intelligent construction model and key methods based on digital twin. In Proceedings of the 2020 Annual Meeting of the Chinese Society of Civil Engineering, Beijing, China, 9 September 2020; China Architechture Publishing \& Media Co., Ltd.: Beijing, China, 2020; Volume 13, pp. 119-131. (In Chinese)

28. Nepal, M.P.; Hon, C.; Lee, J.; Xiang, Z. Towards an Integrated Approach to Infrastructure Damage Assessment in the Aftermath of Natural Hazards. Buildings 2021, 11, 450. [CrossRef]

29. Li, B.; Lee, Y.; Yao, W.; Lu, Y.; Fan, X. Development and application of ANN model for property prediction of supercritical kerosene. Comput. Fluids 2020, 209, 104665. [CrossRef]

30. Yokoyama, A.; Yamaguchi, N. Comparison between ANN and random forest for leakage current alarm prediction. Energy Rep. 2020, 6, 150-157. [CrossRef]

31. Hwang, J.Y.; An, J.G.; Aziz, A.; Kim, J.H.; Jeong, S.M.; Song, J.S. Interworking models of smart city with heterogeneous internet of things standards. IEEE Commun. Mag. 2019, 57, 74-79. [CrossRef]

32. Liu, Z.; Liu, Z.; Sun, J.; Du, X. Intelligent construction methods and model experiments based on digital twins. J. Build. Struct. 2021, 42, 26-36. (In Chinese)

33. Fang, X.; Gebraeel, N.Z.; Paynabar, K. Paynabar Scalable prognostic models for large-scale condition monitoring applications IISE Trans. 2017, 49, 698-710. [CrossRef]

34. Rajesh, P.; Manikandan, N.; Ramshankar, C.; Vishwanathan, T.; Sathishkumar, C. Digital Twin of an Automotive Brake Pad for Predictive Maintenance. Procedia Comput. Sci. 2019, 165, 18-24. [CrossRef] 
35. Liu, Z.; Jiang, A.; Zhang, A.; Xing, Z.; Du, X. Intelligent Prediction Method for Operation and Maintenance Safety of Prestressed Steel Structure Based on Digital Twin Technology. Adv. Civ. Eng. 2021, 2021, 1-17. [CrossRef]

36. Liu, Z.; Shi, G.; Zhang, A.; Huang, C. Intelligent Tensioning Method for Prestressed Cables Based on Digital Twins and Artificial Intelligence. Sensors 2020, 20, 7006. [CrossRef]

37. Zhou, Z.; He, G.; Wang, S.; Jin, G. Subgrid-scale model for large-eddy simulation of isotropic turbulent flows using an artificial neural network. Comput. Fluids 2019, 195, 104319. [CrossRef]

38. Hempert, F.; Boblest, S.; Ertl, T.; Sadlo, F.; Offenhäuser, P.; Glass, C.; Hoffmann, M.; Beck, A.; Munz, C.-D.; Iben, U. Simulation of real gas effects in supersonic methane jets using a tabulated equation of state with a discontinuous Galerkin spectral element method. Comput. Fluids 2017, 145, 167-179. [CrossRef]

39. Sama, A.; Ayoub, K.J. Verifying the performance of artificial neural network and multiple linear regression in predicting the mean seasonal municipal solid waste generation rate: A case study of Fars province, Iran. Waste Manag. 2016, 48, 14-23. [CrossRef]

40. Liu, Z.; Jiang, A.; Shao, W.; Zhang, A.; Du, X. Artificial-Neural-Network-Based Mechanical Simulation Prediction Method for Wheel-Spoke Cable Truss Construction. Int. J. Steel Struct. 2021, 21, 1032-1052. [CrossRef]

41. Liu, Z.; Han, Z.; He, J.; Wang, Z. Sensitive Test on Relaxation of Cable and Reliability Assessment of Spoke Cable-truss Structure. J. Tongji Univ. (Nat. Sci.) 2019, 47, 7. (In Chinese)

42. Ejohwomu, O.A.; Oshodi, O.S.; Oladokun, M.; Bukoye, O.T.; Emekwuru, N.; Sotunbo, A.; Adenuga, O. Modelling and Forecasting Temporal PM2.5 Concentration Using Ensemble Machine Learning Methods. Buildings 2022, 12, 46. [CrossRef] 